\title{
Predictive Optimal Management Method for the control of polygeneration systems
}

\author{
Andrés Collazos, François Maréchal* \\ École Polytechnique Fédérale de Lausanne, LENI-STI, Bât. ME A2, Station 9, CH-1015 Lausanne, \\ Switzerland
}

\begin{abstract}
A predictive optimal control system for micro-cogeneration in domestic applications has been developed. This system aims at integrating stochastic inhabitant behavior and meteorological conditions as well as modeling imprecisions, while defining operation strategies that maximize the efficiency of the system taking into account the performances, the storage capacities and the electricity market opportunities. Numerical data of an average single family house has been taken as case study. The predictive optimal controller uses mixed integer and linear programming where energy conversion and energy services models are defined as a set of linear constraints. Integer variables model start-up and shut down operations as well as the load dependent efficiency of the cogeneration unit. This control system has been validated using more complex building and technology models to asses model inaccuracies and typical demand profiles for stochastic factors. The system is evaluated in the perspective of its usage in Virtual Power Plants applications.
\end{abstract}

Key words: predictive control, optimal management, polygeneration, microcogeneration

\section{Introduction}

The integration of polygeneration systems in urban areas is seen as one of the promising routes for adressing $\mathrm{CO}_{2}$ mitigation needs. For example, decentralized combined heat and power production is foreseen in virtual power plant concepts [1]. The design of polygeneration systems in urban areas relies on the definition of the system management strategy that decides the operation of the energy conversion equipment (cogeneration

\footnotetext{
* Corresponding author.

Email address: francois.marechal@epfl.ch (François Maréchal).
} 
and heat pumping) and of the energy storage system in order to provide the energy services required at minimum cost. The design method is typically based on the definition of average days from which the ambient temperature and the demand profiles are taken as reference. One key component of this strategy is the energy storage equipment that is used to create a phase shift between the energy conversion and the demands allowing for equipment size reduction and better profitability. When the management strategy is based on optimization methods such as presented in Weber et al. [2], the design method relies on the definition of typical days during which the performances are computed assuming a perfect knowledge of the temperature profiles and energy demand. This assumption is, however, not acceptable when developing a management strategy for an existing system since these profiles are stochastic and are not perfectly predictable. The goal of this paper is to present a predictive control strategy for the optimal management of polygeneration systems in complex buildings. The method includes a predictive model of the energy demand of the building based on the prediction of the ambient temperature and an Auto Regressive model with eXternal inputs (ARX) of the building heat losses, combined with a simplified simulation of the heat distribution system. The optimal management strategy uses a mixed integer linear programming model to decide the start up and shut down times of the equipment and decide the heat storage management. The optimal control system thus developed has been validated by connecting it with a detailed building simulation model that is assumed to represent the real non linear and stochastic behavior of the building in its environment.

Finally, access to the electricity market price has been assumed. As targeted in virtual power plants concepts, it has been demonstrated that it is possible to use such systems for exploiting the energy storage systems - including the building structure - to increase the combined heat and power production, thus increasing the benefit of a globalized power production system.

\section{Domestic energy system studied}

The system under study includes one cogeneration unit (a Stirling Engine) and a backup boiler, both fueled by natural gas. The system supplies heat to two heat storage tanks: one for the heating system, the other for the domestic hot water (DHW). The temperature in the heat distribution system (radiator system) is controlled by a 3 -way valve and the temperature set point is determined as a function of the ambient and room temperatures using a heat loss model and heat distribution model.

On Figure 1, $T_{d h w}$ and $T_{s h}$ are the temperatures of the water in the DHW tank and in the heat storage. $T_{i n}$ and $T_{e x t}$ are the room and outside temperatures of the building respectively. $T_{c g, \text { out }}(t)$ is the temperature of the water exiting the cogeneraton unit, $T_{b, \text { out }}(t)$ is the temperature of the water exiting the back-up boiler, $T_{d h w, i n}(t)$ is the temperature of the hot water going into the DHW tank, $T_{s h, i n}(t)$ is the temperature of the hot water going into the heat storage tank and $T_{r}$ is the nominal return temperature of the water. $\dot{m}_{c g}(t)$ and $\dot{m}_{b}(t)$ are the mass flows entering the cogeneration unit and the back-up boiler respectively. $\dot{m}_{s h}(t)$ and $\dot{m}_{d h w}(t)$ are the mass flows sent to the heat storage and the DHW tank. $\dot{E}_{c g}$ is the electrical power output of the cogeneration unit, $\dot{E}_{e g, o u t}$ is the electrical power delivered by the electricity grid, $\dot{E}_{e g, \text { in }}$ is the power sold to the grid and $\dot{E}_{h, i n}$ is the electrical power consumption in the building. 
The independent variables are the load charge of the cogeneration unit $u_{c g}(t)=\frac{\dot{Q}_{c g}(t)}{\dot{Q}_{c g}^{\max }}$, the load charge of the storage heat output $u_{s h}(t)=\frac{Q_{s h}(t)}{Q_{s h}^{\max }}$, the load charge of the back-up boiler $u_{b}(t)=\frac{\dot{Q}_{b}(t)}{\dot{Q}_{b}^{\max }}$, and the 3-way valve control $u_{v l v}(t)=\frac{Q_{s h}(t)}{\dot{Q}_{c g}(t)+\dot{Q}_{b}(t)}$.

Here $\dot{Q}_{c g}(t), Q_{s h}(t)$ and $\dot{Q}_{b}(t)$ are the heat outputs at time $t$ of the cogeneration unit, the heat storage tank and the boiler respectively. The superscript max indicates the maximum allowed value for each variable.

The sizes of the units in the system are calculated using a the Queuing Multi Objective Optimizer (QMOO) developed at the Energy Systems Laboratory at the EPFL (Leyland [3]) in combination with a linear programming problem as described by in Weber et al. [2]. The sizes of the units considered are shown on Table 1.

Table 1

Unit characteristics. $\dot{Q}=$ maximum heat output, $\eta=$ efficiency, $\dot{E}=$ maximum electrical output, $\eta_{e l}=$ electrical efficiency, $\eta_{t h}=$ thermal efficiency, $V=$ volume

\begin{tabular}{|c|c|c|c|c|c|c|c|c|}
\hline \multicolumn{2}{|c|}{ Boiler } & \multicolumn{4}{|c|}{ Cogeneration Engine } & \multicolumn{2}{|c|}{ Heat Stroage } & \multirow{2}{*}{$\frac{\text { DHW tank }}{V\left[m^{3}\right]}$} \\
\hline$\dot{Q}[k W]$ & $\eta[-]$ & $\dot{E}[k W]$ & $\eta_{e l}[-]$ & $\dot{Q}[k W]$ & $\eta_{t h}[-]$ & $V\left[m^{3}\right]$ & $\dot{Q}[k W]$ & \\
\hline 2.17 & 0.8 & 2.25 & $0.2-0.25$ & 6.83 & $0.7-0.75$ & 0.45 & 10 & 0.12 \\
\hline
\end{tabular}

The building characteristics correspond to the sIA $380 / 1$ target value single family home described by Dorer et al. [4]. The size of the cogeneration unit corresponds to an overall full load operating time of 3962 hours per year. The (variable) efficiencies used are based on the manufacturer's technical data [5].

\section{The predictive controller}

The predictive control strategy calculates the optimal values of the controlled variables for $t_{0} \leq t \leq t_{0}+\Delta t_{M H}$, where $t_{0}$ is the current instant and $\Delta t_{M H}$ is the moving horizon length. The strategy is re-evaluated after every time step $\Delta t$. The optimal strategy is calculated by solving a Mixed Integer and Linear Programming (MILP) model of the system. The objective of the MILP is to minimize the sum of the operating costs as well as a penalty term that measures the total time during which the room temperature is outside the comfort range, for the given time horizon. In order to give a priority to comfort, a significant relative weight is assigned in the objective function to comfort violations. The operating costs are the sum of the gas consumption in the cogeneration unit and back-up boiler, added to the electricity consumption minus the electricity export. The back up boiler is modeled with a constant efficiency. The losses in the storage tanks are modeled using standard heat loss equations. The minimum required temperature for space heating water is calculated using the normalized equation from siA [6] applied to the nominal outlet temperature $T_{\text {ext }, 0}$ and the nominal heating water supply temperature $T_{\text {min,0 }}[7]$. The room temperature of the building is calculated by a second order ARX model with the space heat delivered as input

$$
\Delta T_{i n}\left(t+2 \Delta t_{s}\right)+a_{1} \Delta T_{i n}\left(t+\Delta t_{s}\right)+a_{2} \Delta T_{i n}(t)=b_{1} \dot{Q}_{h, i n}\left(t+\Delta t_{s}\right)+b_{2} \dot{Q}_{h, i n}(t)
$$

where $\Delta T_{i n}(t)=T_{i n}(t)-18$ and $T_{i n}(t)$ is the room temperature. $a_{1}, a_{2}, b_{1}$ and $b_{2}$ are the coefficients of the model, $\dot{Q}_{h, i n}(t)$ is the heat input and $\Delta t_{s}=\frac{1}{4} \Delta t$. 
The control variable for the cogeneration unit is the heat output given by

$$
\begin{aligned}
\dot{Q}_{c g}(t) & =u_{c g}(t) \cdot \dot{Q}_{c g}^{\text {max }}, \\
\dot{Q}_{c g}^{\text {min }} \cdot c g_{o n}(t) & \leq \dot{Q}_{c g}(t) \leq \dot{Q}_{c g}^{\text {max }} \cdot c g_{o n}(t)+c g_{\text {start-up }}(t) \cdot \eta_{c g, t h}^{\text {start }-u p} \cdot \dot{Q}_{c g, g a s}^{\text {max }} .
\end{aligned}
$$

The electricity output of the cogeneration unit as a function of the heat output is given by the following piece-wise function

$$
\begin{aligned}
\dot{E}_{c g}(t)= & c g_{o n} \cdot \dot{E}_{c g}^{m i n}+c g_{s t a r t-u p}(t) \cdot \eta_{c g, e l}^{s t a r t-u p} \cdot \dot{Q}_{c g, g a s}^{\max } \\
& +c g_{p w}\left[m_{e l, 1}\left(\dot{Q}_{c g}(t)-\dot{Q}_{c g}^{\text {min }}\right)+\dot{E}_{c g}^{\text {min }}\right] \\
& +\left(1-c g_{p w}(t)\right)\left[m_{e l, 2}\left(\dot{Q}_{c g}(t)-\dot{Q}_{c g, p w}\right)+m_{e l, 1}\left(\dot{Q}_{c g, p w}-\dot{Q}_{c g}^{m i n}\right)\right]
\end{aligned}
$$

The first term of the right hand side of Equation 4 corresponds to the minimal electricity output. $c g_{o n} \in\{0,1\}$ is the integer variable that indicates if the cogeneration unit is on or off. The second term corresponds to the electricity output when the unit is started. The variable $c g_{\text {start-up }}(t)$ indicates whether the cogeneration unit has been started at time $t$; note that this is not an integer variable, but it can only take the values 0 and 1 because of its definition. The third and fourth terms of Equation 4 correspond to two piecewise linear components modeling the electrical output as a function of the heat output $\dot{Q}_{c g}(t) . m_{e l, 1}$ and $m_{e l, 2}$ are the linear slopes, $c g_{p w} \in\{0,1\}$ is an integer variable that indicates which piece is used and $\dot{Q}_{c g, p w}$ is the breakpoint. A similar equation to Equation 4 is used for modeling the gas input as a function of the heat output. Finally, the cogeneration unit is constrained to stay on for at least $n_{c g, o n}^{\min }$ hours.

In order to add the knowledge of the periodicity of one day to the next when calculating the strategies, a "cyclic" constraint is included

$$
A\left(t_{0}+24+1\right)=A\left(t_{0}+1\right)
$$

where $A$ is any state variable such as the room temperature, the energy stored in the heat storage tanks or the controlled variables. $t_{0}$ is the time at which the strategy is being calculated. The cyclic constraint (Equation 5) is applied at time $t_{0}+1$ in case the state at time $t_{0}$ is not within the desired temperature range (in case of a large perturbation or a big discrepancy in the predictions). It is assumed that the system can move to a "good" state within an hour.

\section{Validation}

In order to validate this control strategy a numerical model of the installation has been used. This consists of a SiMULINK model of the building's thermal behavior adjusted to correspond to the SIA 380/1 target value of a single family home [4], of the heat distribution system and a non linear cogeneration engine using the efficiency charts on [5]. The models described in Section 3 for the heat storage and DHW tank were used also for the simulation of these units. Standard and stochastic profiles of outside temperature, solar gains, internal free gains, electricity consumption and DHW consumption were used to simulate the environment and the inhabitants' behavior. The temperature was predicted by its mean value from the past 30 days as described by [8]. The same approach was used to predict the solar gains, electricity consumption and DHW consumption. The gains from inhabitants and electrical appliances were considered as perturbations since they 
are not easily measured in a real implementation. The simulation is performed using the real values to validate the behavior of the control system when there are discrepancies between the predicted and real values. A correction is applied when the stored energy or the temperature of the storage tanks are outside the allowed range. This correction consists of adding some energy (positive or negative) in order to keep the allowed range.This extra energy gives an estimation of the reserve needed for the storage tanks.

\section{Results}

The MILP optimization was performed using AMPL-CPLEX. The calculation times were below 1 minute per strategy evaluation. The controller was applied during five days in spring. The operating costs for the system with the cogeneration unit are $13 \%$ lower than the operating costs when all the heat input to the system is delivered by a boiler and when all the elecricity is bought from the grid, with the same energy storage and distribution strategy. Figure 2 compares the room temperature $T_{i n}$ with its set-point and the temperature predicted by the controller for three non consecutive strategy reevaluation times. This picture shows that the controller reevaluates the strategy and adapts it at every time interval allowing it to compensate for the perturbations and for inaccuracies in the predictions. The Figure also shows that the predicted strategy differs from the final strategy for further times in the horizon (oscillations of $u_{c g}$ around 1160-1170), thus the necessity to re-evaluate the strategy more often than the actual horizon length.

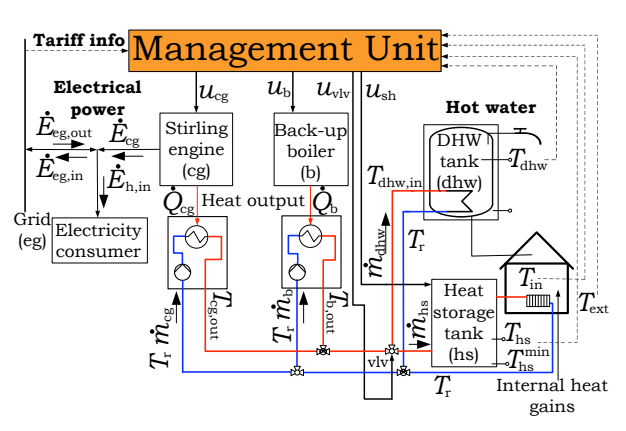

Figure 1. Test case system

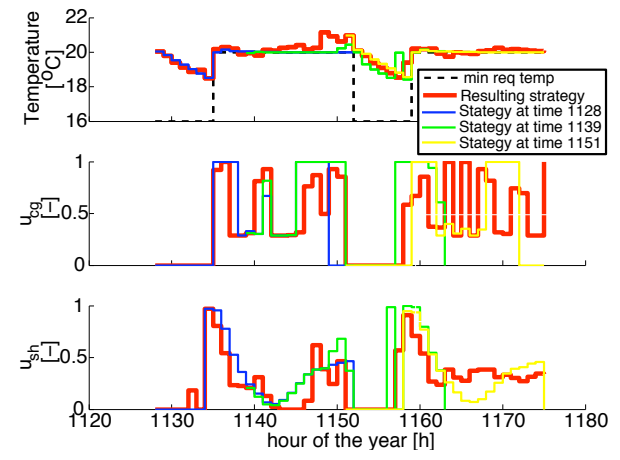

Figure 2. Resulting and predicted strategies

Figure 3 shows the energy management with and without the cyclic constraint (Equation 5). The strategy that uses the cyclic constraint features a better management of the storage tanks, preventing a storage of heat in the tanks for longer periods and therefore reducing the storage losses. The operating costs for this strategy are around $2 \%$ lower with no extra penalty in the comfort or in the reserve energy required (Section 4).

In the Virtual Power Plants perspective [1], the case where the electricity price is not constant has also been assessed to demonstrate the capabilities of the controller to adapt the strategy to take advantage of a time dependent electricity market price. Figure 4 compares the strategies with a varying electricity price and a constant electricity price. The varying price reduces the cost of energy supply by $5 \%$ with no additional comfort violation. The constant electricity price is the average of the varying electricity price over the 5 days considered. 


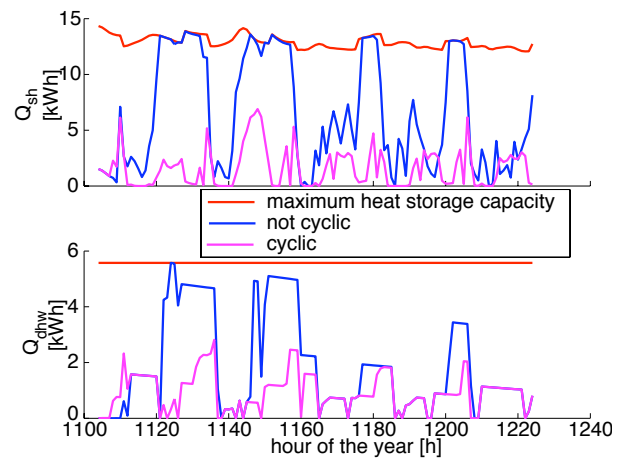

Figure 3. Stored energy with and without the cyclic horizon approach

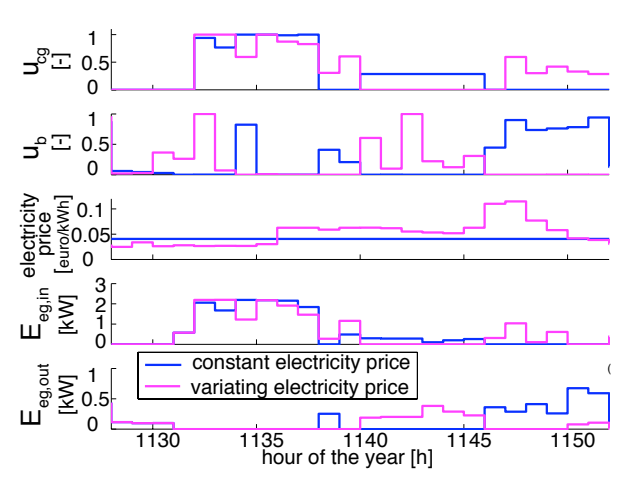

Figure 4. Time dependent electricity price

\section{Conclusions}

A model based predictive controller has been developed using a Mixed Linear and Integer Programming model to define the optimal management strategy of micro-cogeneration systems in building applications. The MILP model takes into account starting and shutdown of the unit as well as the partial load efficiency using a piecewise formulation. The model includes the balance of the hot water storage tanks as well as the heat accumulation in the building envelope.

The controller was validated with a numerical model of the system that is more detailed than the model used for the predictive controller. The predictions of temperature and solar gains as well as the consumption of domestic hot water and electricity are obtained. The cyclic horizon has proved to deliver a better performance than the "open" horizon.

In the virtual power plants perspective, this controller shows an ability to adapt the strategy in order to profit from fluctuating price of the electricity.

\section{References}

[1] System-development, build, field installation and european demonstration of a virtual fuel cell power plant, consisting of residential micro-chps.

[2] C. Weber, F. Maréchal, D. Favrat, S. Kraines, Optimization of an SOFC-based decentralized polygeneration system for providing energy services in an office-building in Tokyo, Applied Thermal Engineering 26 (13) (2006) 1409-1419.

[3] G. Leyland, Multi-objective optimisation applied to industrial energy problems, Ph.D. thesis, EPFL, Lausanne, Switzerland (2002).

[4] V. Dorer, R. Weber, A. Weber, Performance assessment of fuel cell micro-cogeneration systems for residential buildings, Energy and Buildings 37 (2005) 1132-1146.

[5] Solo stirling 161 combined power / heat (chp) module (2005).

[6] Standard sia 384/2 (1988).

[7] M. Zehnder, Efficient air-water heat pumps for high temperature lift residential heating, including oil migration aspects, Ph.D. thesis, École Polytechnique Fédérale de Lausanne (2004).

[8] G. Henze, D. Kalz, C. Felsman, G. Knabe, Impact of Forecasting Accuracy on Predictive Optimal Control of Active and Passive Building Thermal Storage Inventory, HVAC \& R Research 10 (2) (2004) 153-178. 